\title{
Translational target for checkpoint inhibitors
}

Immune evasion by cancer cells through activation of inhibitory checkpoints has become a key focus for targeted therapies. Notably, therapeutic antibodies that block the interaction between the immune checkpoint programmed cell death 1 ligand 1 (PD-L1) and its receptor, PD-1, are approved for several cancer types. However, the lack of a biomarker to predict treatment response, as well as the development of resistance, limit the utility of such therapies. Now, a study in Nature Medicine has shown that the eukaryotic translation initiation complex eIF4F is an upstream regulator of PD-L1 expression and a promising target for improved immunotherapies.

The eIF4F complex is an important part of the translational machinery and a promising anticancer target owing to its position downstream of several oncogenic signalling networks. In the current study, Cerezo et al. sought to discover whether $\mathrm{PD}-\mathrm{L} 1$ expression on tumour cells could be under translational control by eIF4F. They focused on interferon- $\gamma($ IFN $\gamma)$-induced PD-L1 upregulation as this is a classic immune evasion response of tumour cells to infiltrating IFN $\gamma$-secreting

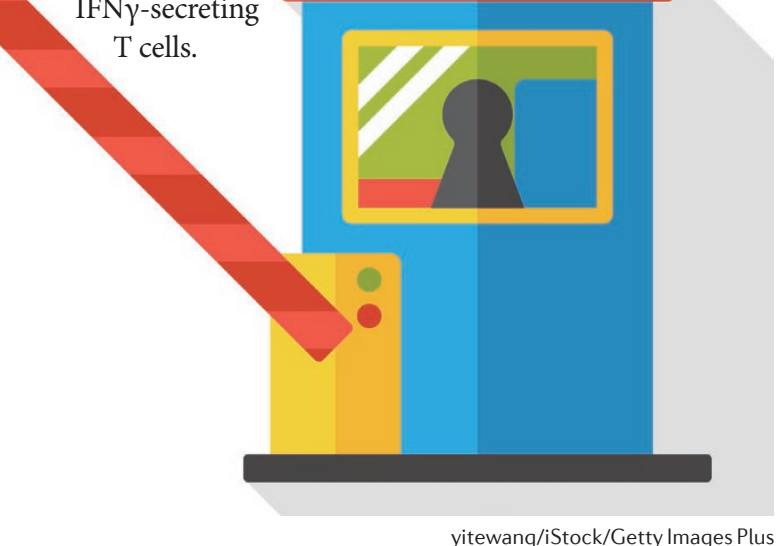

elF4F is an IFN $\gamma$-induced expression of PD-L1 upstream in cancer cell lines. Application of the regulator eIF4F inhibitor silvestrol significantly blunted the upregulation of PD-L1 expression seen in response to IFN $\gamma$ in expression and a promising target for improved immunotherapies melanoma, breast and colon tumour cell lines. Moreover, melanoma cell lines derived from transgenic mice with increased eIF4F complex formation showed enhanced IFN $\gamma$-induced PD-L1 surface expression.

To extend their findings to human tumours, the investigators analysed tumour samples from 59 patients with metastatic melanoma, and found a significant correlation between PD-L1 surface expression, $\mathrm{CD}^{+} \mathrm{T}$ cell density and eIF4F complex formation. The patients had all received treatment with pembrolizumab, a PD-1-targeted monoclonal antibody, and response to treatment correlated with the level of eIF4F activation. The authors suggest eIF4F complex formation could therefore represent a theranostic biomarker to predict response to PD-1 blockade.

Next, Cerezo et al. turned to the mechanistic link between eIF4F inhibition and PD-L1 downregulation. Quantitative real-time PCR indicated that blockade of eIF4F with silvestrol dampened inducible PD-L1 expression via an effect on $P D-L 1$ gene transcription.

So how does blockade of translational machinery lead to downregulation at the transcriptional level? The researchers used mRNA profiling and microarray analysis to identify mRNA species in melanoma cell lines that were transcriptionally upregulated by IFN $\gamma$ and translationally downregulated by silvestrol.
The analysis revealed that signal transducer and activator of transcription 1 (STAT1) mRNA provides the missing link between eIF4F activity and $P D-L 1$ transcription. In line with these findings, silvestrol dosedependently decreased STAT1 protein levels, and melanoma cells from transgenic mice with constitutively upregulated eIF4F activity expressed higher levels of STAT1 protein compared with melanoma cells with normal eIF4F activity.

The authors concluded that activation of eIF4F in response to immune cell-derived IFN $\gamma$ increases translation of STAT1 mRNA, elevating the level of this transcription factor and thereby promoting PD-L1 expression and immune evasion by the tumour.

In immunocompetent mice harbouring melanoma allografts, daily intraperitoneal injection with silvestrol significantly reduced tumour growth and was associated with increased immune cell infiltration of the tumour. Importantly, the same dose of silvestrol had no effect in nude mice, underscoring the key role of the immune system in the drug response.

eIF4F inhibitors have gained increasing attention as potential cancer therapies owing to their direct antitumour effects. The current study suggests that these molecules have an additional, immune-mediated mode of action that might improve immunotherapy efficacy and provides a tractable target for small-molecule intervention.

Katie Kingwell

ORIGINAL ARTICLE Cerezo, M. et al.

Translational control of tumor immune escape via the elF4F-STAT1-PD-L1 axis in melanoma. Nat. Med. https://doi.org/10.1038/s41591-018-0217-1 (2018) 